\title{
Transfer Across Perfused Human Placenta. II. Free Fatty Acids
}

\author{
Joseph Dancis ${ }^{[35]}$, Valerie Jansen, Herbert J. Kayden, \\ Henning Schneider, and Mortimer Levitz \\ Departments of Pediatrics, Obstetrics and Gynecology, and Medicine, New York University \\ School of Medicine, New York, New York, USA
}

\section{Extract}

The rate of transfer of palmitic acid across human placenta was measured in an in vitro perfusion system. The rates of transfer from maternal to fetal circulation and in reverse direction were about the same, and were considerably less than those for antipyrine and leucine. The transfer rate for linoleic acid was similar to that for palmitic acid. An estimate of the absolute rate of transfer for free fatty acids was made which yielded a figure of $6.8 \mathrm{mmol} / 24 \mathrm{hr}$. which is less than that required for the deposition of fat by the fetus in the last trimester. These calculations indicate that during the period of rapid accumulation of fat the fetus converts glucose and/or amino acids to that purpose.

Uptake and metabolism of palmitate by placental slices differs significantly in the human and the guinea pig.

\section{Speculation}

It has been suggested that the placental capacity for transfer of some nutrients greatly exceeds requirements, whereas with respect to other nutrients, the capacity for transfer is far more limited. A general reduction in placental function may produce selective nutritional deficiencies in the fetus, beginning with those factors transferred with the smallest margin of safety.

\section{Introduction}

The transport of maternal lipids to the fetus and their contribution to the fetal economy have been investigated in several species $[4,6,8,10,11,15,20,21]$. However, there is no information concerning the human except for a brief statement that radioactive palmitate administered to two women just before therapeutic abortion appeared to be transferred rapidly across the placenta [20].

The recent development of an in vitro perfusion technique suitable for the study of transport across the human placenta [17] has permitted the orderly investi- gation of a series of nutrients. In the present study, the rates of transfer for palmitic acid and linoleic acid are reported as representative of free fatty acids (FFA). The rates of transfer were determined simultaneously for antipyrine and leucine, which permitted some inferences concerning placental function and the availability of maternal fatty acids for fetal nutrition. The results are compared with those obtained previously for the guinea pig using in situ perfusion of placenta. The observed differences were explored further using in vitro techniques with slices of guinea pig and human placenta. 


\section{Methods}

\section{Perfusion}

The perfusion technique has been described in detail previously [17]. In brief, placentas are obtained at term after delivery by caesarian or per vaginam and rapidly chilled in buffer at $5^{\circ}$. The fetal vessels to a cotyledon weighing approximately $20 \mathrm{~g}$ are cannulated and perfused with oxygenated buffer solution. The cotyledon is isolated and a maternal circuit is established by piercing the decidual plate with two small glass cannulas so that they penetrate the intervillous space. The perfusion is continued in a chamber maintained at $37^{\circ}$. In the experiments to be described, both maternal and fetal circuits were kept open; that is, the perfusate was not recirculated. Both maternal and fetal perfusates were equilibrated with $95 \%$ oxygen$5 \%$ carbon dioxide.

\section{Perfusate}

Most of this series of experiments were conducted with serum as perfusate to permit solubilization of the free fatty acids by binding to the serum proteins. An unidentified, nondialyzable factor in the serum frequently caused vasoconstriction in the fetal circuit, manifested by hypertension and reduced blood flow. The complication was eliminated or reduced by diluting the serum with Earle's buffered salt solution, $1: 1$ or $1: 2$.

Outdated plasma containing citric acid-dextrose is dialyzed against 30 volumes of isotonic saline at $4^{\circ}$ overnight. The dialyzed plasma is stored frozen until ready for use. Before perfusion, double strength Earle's buffered salt solution is added in equal volumes; allowance is made for the content of $\mathrm{NaCl}$ in the dialyzed plasma. Then $\mathrm{CaCl}_{2}$ is added in excess $(0.04 \mathrm{~g} / 100 \mathrm{ml})$ to make certain that clotting is completed. The clots are removed by filtration and penicillin and streptomycin, 5,000 units each $/ \mathrm{ml}$, are added. The $\mathrm{pH}$ is adjusted to 7.4 with $6 \mathrm{~N} \mathrm{HCl}$.

Isotopes and fatty acids are added to $2 \mathrm{ml}$ prepared perfusate $12 \mathrm{hr}$ before perfusion to ensure completion of protein binding. Fat-free $(<0.005 \%)$ bovine albumin [24] was used in $1 \%$ concentration in Earle's buffered salt solution in some experiments.

\section{Determination of Radioactivity}

In perfusion experiments with antipyrine- $N$ methyl- ${ }^{3} \mathrm{H}$, palmitic acid-1-14 $\mathrm{C}$, and $\mathrm{L}$-leucine-4, $5-{ }^{3} \mathrm{H}$, the first two compounds were extracted from the per- fusate with chloroform. Two milliliters of perfusate were extracted four times with $2 \mathrm{ml}$ chloroform. The chloroform phase was transferred to a counting vial and was evaporated to dryness. Diotol scintillant [7] was added to determine radioactivity. The two isotopes were assayed in a scintillation spectrometer [25]. The water phase was transferred directly to Diotol for assay of tritiated leucine.

\section{Uptake by Placental Slices}

Guinea pig placentas were obtained by hysterotomy from pregnancies estimated to be within 2 weeks of term. Human placentas were obtained from elective caesarian sections or from normal deliveries at term. Tissue slices were prepared with a Stadie-Riggs slicer. About $100 \mathrm{mg}$ tissue were placed in $2 \mathrm{ml}$ plasma-Earle's solution, $1: 1, \mathrm{pH} 7.4$, containing palmitic acid-1-14 $\mathrm{C}$, were gassed with $95-5 \% \quad \mathrm{O}_{2}-\mathrm{CO}_{2}$, and were incubated with shaking at $37^{\circ}$ on a Dubnoff metabolic shaker. After incubation, the slices were removed, rinsed quickly in chilled Earle's solution, blotted dry on paper toweling, and weighed in counting vials. Tissue solubilizer, $0.75 \mathrm{ml}$ [26], was added to the vial and the contents were incubated at $37^{\circ}$ overnight. Radioactivity was determined in a scintillation counter in toluene scintillant and is reported as counts per minute per gram, wet wt.

\section{Thin Layer Chromatography}

Thin layer chromatography was carried out on precoated Silica Gel G plates [27] in a solvent system of petroleum ether-ethyl ether-glacial acetic acid, $90: 10: 1$. Chloroform extracts, obtained after Folch extraction of the perfusate, along with appropriate standards, were placed at the lower margin of the plates. Separation was achieved by ascending chromatography and the individual lipids were identified by the position of the standards. The gel containing the lipids was scraped directly into counting vials, scintillant solution was added, and counting was done. Quench correction curves were obtained by means of an automatic external standard and disintegrations per minute were calculated from activity of known standards.

\section{Reagents and Isotopes}

The labeled substrates were obtained from New England Nuclear [28] as follows: palmitic acid- $-{ }^{14} \mathbf{C}$, $0.05 \mathrm{mCi} / 1.3 \mathrm{mg}$, palmitic acid-(N)- $, 10-{ }^{3} \mathrm{H}, 5.0 \mathrm{mCi} /$ 
Table I. Transfer rate of leucine and palmitic acid relative to antipyrine

\begin{tabular}{lcc}
\hline & Leucine & Palmitic acid \\
\hline Maternal to fetal transfer & & \\
Experiment 1 & 59 & 11 \\
Experiment 2 & 48 & 4 \\
Fetal to maternal transfer & & \\
Experiment 3 & 44 & 11 \\
Experiment 4 & 31 & 6
\end{tabular}

1 The clearances of radioactive an tipyrine, leucine, and palmitic acid were measured in each direction, toward the fetal and toward the maternal circulations, in four experiments. Results are presented as a percentage of antipyrine clearance.

$5.6 \mathrm{mg}$, linoleic acid-1 $1{ }^{14} \mathrm{C}, 0.05 \mathrm{mCi} / 0.25 \mathrm{mg}$, L-leucine$4,5-{ }^{3} \mathrm{H}(\mathrm{N}), 0.25 \mathrm{mCi} / 0.25 \mathrm{mg}$, antipyrine- $N$-methyl ${ }^{3} \mathrm{H}$, $0.25 \mathrm{mCi} / 3 \mathrm{mg}$. L-Leucine was obtained from Schwarz/ Mann [29], and palmitic and linoleic acids from Sigma Chemical Company [24].

\section{Results}

The transfer rates for antipyrine, leucine, and palmitic acid were measured simultaneously in four experiments, two from maternal to fetal circulation and two in the reverse direction (Table $\mathrm{I}$ ). The results are presented as a percentage of the antipyrine rate of transfer. The transfer rates for palmitic acid are low and about the same in both directions.

Direct comparison of palmitic and linoleic acids in a series of experiments revealed only a slight difference in transfer rates. The ratios of transport rates of palmitic to linoleic acids in three experiments were $1.11,1.14$, and 1.08 .

In the experiments described so far, human serum containing endogenous FFA in the same concentration was used as maternal and fetal perfusate. The movement of the tracer-labeled FFA, therefore, measured exchange rates rather than net transfer. To determine whether the exchange rates were comparable with the potential for net transfer, two experiments were done with a $1 \%$ perfusate of commercially available, fatfree bovine serum albumin. In both experiments two levels of palmitic acid-1-14 $\mathrm{C}$ were added to the maternal circulation in sequence, trace amounts $(0.03 \mathrm{mg} / 100$ $\mathrm{ml}$ ) and an amount roughly equivalent to $25 \%$ of the endogenous level (l mg/100 ml) to correct for the reduced concentration of albumin used in the perfusion. Antipyrine was also added to the maternal perfusate. The transfer rates for the low and high levels of palmitic acid were 2 and $1.6 \%$ of the antipyrine transfer rate in the first experiment and 6.5 and $5 \%$, respectively, in the second experiment. The transfer rates at the high concentrations of palmitic acid, now representing net transfer rather than exchange, are somewhat lower but of the same order of magnitude as the results with serum, possibly because of more avid binding by fat-free albumin [1]. The radioactivity in the fetal perfusate was identified as primarily FFA by thin layer chromatography (100\% in one experiment, and over $70 \%$ in the second). The similarity in percentage of transfer in each experiment between low and high levels of palmitic acid excluded mixing and exchange with any significant pools of palmitic acid.

In a previous study, the rate of transfer of palmitic acid across guinea pig placenta had been investigated with an in situ perfusion technique [10] with results considerably at variance with those presented here for the human placenta. The rates of uptake of palmitic acid by placental slices from the two species were measured to determine whether, under identical conditions, the handling of palmitic acid did differ. The relatively flat curves at $0^{\circ}$ and $37^{\circ}$ with guinea pig placenta indicated little metabolism of FFA. The slight peak at $30 \mathrm{~min}$ in one experiment was not investigated further. The higher levels of uptake at $37^{\circ}$ could reflect changes in protein binding in the placenta or incubation medium. However, in the human, the progressive uptake with time at $37^{\circ}$ contrasts sharply with performance at $0^{\circ}$, which indicates extensive metabolism of palmitic acid.

This presumption was confirmed by thin layer chromatography of the radioactivity extracted from placenta at $60 \mathrm{~min}$. In guinea pigs, over $98 \%$ of the radioactivity migrated with FFA; in humans, only $42 \%$ remained as FFA; $30 \%$ and $19 \%$ were converted to phospholipids and triglycerides, respectively. There was relatively little combustion to $\mathrm{CO}_{2}$ (about $1 \%$ ), a result similar to those obtained by others [16]. Since these studies have been done, a more extensive study of the metabolism of palmitic acid by human placental and fetal tissues has appeared [23].

\section{Discussion}

Antipyrine is very rapidly transferred across membranes. The amount transferred across the placenta per unit of time is largely dictated by the circulatory factors controlling the amount delivered to one side of the placental membrane and the efficiency with which it is removed from the other side. This behavior indicated that the transfer rate for antipyrine might serve 
as a useful base line for comparing transfer rates for other materials by correcting for such variables. Our experience so far supports this thesis, in that such ratios have proved more consistent within any one experiment than absolute rates of transfer.

Transfer of palmitic acid across the placenta is relatively slow, averaging about $8 \%$ the transfer rate of antipyrine, and is about the same in both directions (Table I). The transfer of leucine, assisted by an active transport mechanism, is considerably faster and appears to be slightly more rapid towards the fetus, which is consistent with the establishment of a small gradient in that direction.

Szabo et al. [19] have studied the transport of palmitic acid across human placenta using the instrumentation originally described by Krantz et al. [12]. Although the perfusion system described by Krantz et al. is the same in principle as the one used in our laboratory, there are important differences in detail, which have been discussed previously [17]. In the present study, a single cotyledon is perfused instead of the entire placenta; this permits the careful selection of a nontraumatized area. The perfusion of the fetal vessels is initiated first and then the maternal circulation is established by inserting cannulas carefully into the appropriate portion of the intervillous space. This contrasts with the nonselective perforation of the decidual plate by 100 polyethylene catheters in the studies described by Szabo et al. [17]. Their estimate of 15-50 $\mu \mathrm{mol}$ palmitic acid $/ \mathrm{hr}$ for the transfer rate for palmitic acid is less than $20 \%$ of the figure derived from our studies (see Appendix), which indicates a much less efficient perfusion system. Antipyrine served as a monitor of the efficiency of the perfusion system in the present studies, and provided a base line in measurement of transfer rates for other substrates. Szabo et al. also reported a more rapid transfer of palmitic acid toward the fetus than in the reverse direction, an observation not confirmed in the present studies.

The relative rates of transfer for a series of materials across human placenta using antipyrine as base line are presented in Table II. For comparison, a similar but not strictly comparable record has been compiled from data collected in this laboratory for transfer across guinea pig placenta. The studies on placental transfer in the guinea pig were undertaken with an in situ perfusion technique $[2,3,10,13,14]$, and measurements were made in the reverse direction, from fetal to maternal circulation. Antipyrine was not used regularly as a base line, so that many of the figures repre- sent rough estimates done at different times in differ-
Table II. Transfer rates across human and guinea pig placental

\begin{tabular}{lcc}
\hline & Guinea pig & Human \\
\hline Sodium & 35 & 25 \\
Amino acids & $30^{2}$ & $50^{2}$ \\
Estriol & 35 & 65 \\
Palmitic acid & 65 & 8
\end{tabular}

1 Transfer rates are presented as a percentage of antipyrine clearance. The experiments with guinea pigs were performed with the in situ perfusion technique measuring transfer from fetus to mother. The experiments with humans measured transfer rates in the reverse direction.

${ }^{2}$ The amino acids studied were $\alpha$-aminoisobutyric acid in the guinea pig and L-leucine in the human.

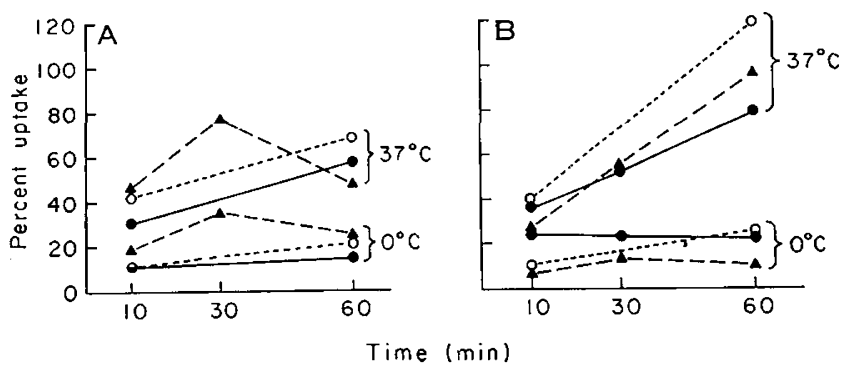

Fig. 1. Uptake of palmitic acid-1-14 $\mathrm{C}$ by placental slices. [(Counts per minute per gram of placenta)/(counts per minute per milliliter of incubation medium) $] \times 100=$ percentage of uptake. $A$ : Quinea pig; B: human.

ent animals. If these limitations are accepted, it can be seen that the transfer of palmitic acid is evidently grossly different in the two species.

In a previous study of amino acid transport [3], the measurement of uptake of $\alpha$-aminoisobutyric acid by placental slices proved to be a useful technique. Although there were many similarities between guinea pig and human placenta, only the latter could be demonstrated to be responsive to insulin. In the present study, the uptake of palmitic acid by placental slices was investigated to determine whether, under highly controlled conditions, one could also detect differences between the two species. A striking difference was easily demonstrable, which reflected the rapid conversion of palmitic acid to complex lipids by human placenta (Fig. 1).

These two observations underscore a feature that must be emphasized constantly in comparative placentology. The guinea pig placenta is similar to the human placenta, as it has very few layers of tissue interposed between the maternal and fetal circulations, thus making it favored by some investigators for study of placental transfer. However, the placenta is an organ that has shown remarkable diversity in ful- 
filling its basic functions, and it is not possible to be certain of the performance of human placenta from studies made in other animals.

The initial objective in establishing the perfusion technique in this laboratory was to investigate relative rates of transfer of a series of nutrients, and to study the mechanisms of transport. The artificial nature of the maternal circulatory system appeared to prohibit the determination of rates of transfer which would have direct relevance to in vivo transfer rates. However, in a previous study [17], the transfer rates for sodium and leucine were reasonably consistent with the limited information concerning in vivo performance. The exchange rate for sodium compared closely with results obtained with in vivo studies in the human and monkey. The transfer of leucine was estimated to be about 3 times the amount required for deposition of protein, which provided a small "safety factor" and possibly an excess for production of energy or conversion to other body constituents.

We have, therefore, been encouraged to apply similar calculations to the transfer of palmitic and linoleic acids, again with results that are consistent with information derived from other sources (see Appen$d i x)$. The human fetus deposits fat at a rapid rate during the last months of pregnancy. The calculations indicate that only $20 \%$ of the estimated requirement for fatty acids is satisfied by placental transfer, the rest requiring de novo synthesis by the fetus.

Such a limitation in supply is consistent with studies of lipid composition and the resultant speculations by Hirsch et al. [9]. In human infants born about 2 months prematurely, the fatty acid composition of the lipid is very similar to that of the mother, which suggests maternal origin of the fatty acids. However, at term after a period of rapid accumulation of fat, the linoleic acid concentration of the lipids drops from $12 \%$ to $1.3 \%$. Linoleic acid is an essential fatty acid which the mother obtains from dietary sources and which the fetus must receive from the mother. If the fetus were forced to synthesize its own fats from other sources, a fat that is poor in linoleic acid would result. If one assumes that the fetus synthesizes $80 \%$ of its fats during this period, as indicated by our studies, one would anticipate a drop in linoleic acid concentration of about $75 \%$. This approaches reasonably closely the observed figure when one takes into account the relatively crude estimates involved in the calculations.

Sheath et al. [18] have estimated fetal extraction of FFA from umbilical arteriovenous differences of samples obtained at delivery. They also conclude that the
FFA supplied to the fetus are insufficient in amount for fat deposition, but suggest that about $80 \%$ of the required amount could be satisfied by placental transfer. Single point analyses of a labile substance like FFA, taken during the stressful period of delivery, provide a very uncertain indication of transfer rates during the last trimester of pregnancy. The derived figure is difficult to reconcile with the observations of Hirsch [9], unless the fetus degrades maternally derived FFA preferentially and synthesizes its own fatty acids de novo.

The FFA plasma concentration in the human infant is lower at term than in the mother. Three theoretical possibilities may be considered. (l) The placenta may establish a gradient as it does for amino acids. This is difficult to exclude with certainty, but there is no supporting evidence. (2) There may be a difference in protein binding offered by maternal and fetal albumin. We are studying this possibility currently. (3) The utilization by the fetus may exceed maternal supplies. Evidence has just been presented which supports this thesis. It may be significant that, in the rabbit, in which species the fetus accumulates very little fat, there is no gradient between maternal and fetal circulation [20].

\section{Appendix}

\section{Estimate of Transfer of FFA to Fetus}

$\begin{aligned} \text { Clearance of antipyrine }= & 3.0 \mathrm{ml} / \mathrm{min}[30] \\ & 0.08 \\ \text { Clearance of palmitic acid }= & 0.24 \mathrm{ml} / \mathrm{min} \text { or } 345 \mathrm{ml} / 24 \mathrm{hr}\end{aligned}$

Approximately $1 / 30$ of placenta is perfused:

$345 \mathrm{ml} \times 30=10,350 \mathrm{ml}$ cleared by placenta $/ 24 \mathrm{hr}$

Assuming that all FFA are cleared at same rate as palmitic acid and linoleic acid:

Maternal level

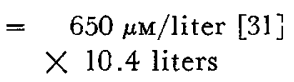

$6760 \mu \mathrm{mol}$ FFA transferred $/ 24 \mathrm{hr}$

Average molecular weight of FFA

$\frac{250}{1.7} \mathrm{~g} \mathrm{FFA}$ transfer-
$\times 30 \quad \mathrm{red} / 24 \mathrm{hr}$
$\frac{51 \mathrm{~g} / \text { month }}{}$

Correcting for esterification with glycerol and water content, this could yield $70 \mathrm{~g}$ fat or about $20 \%$ 
of the approximately $350 \mathrm{~g}$ fat accumulated in the last month of pregnancy [22].

\section{References and Notes}

1. Dancis, J., Jansen, V., And Levirz, M.: Unpublished observations.

2. Dancis, J., ANd Money, W. L.: Transfer of sodium and iodo-antipyrine across guinea pig placenta using an in situ perfusion technique. Amer. J. Obstet. Gynecol., 80: 215 (1960).

3. Dancis, J., Money, W. L., Springer, D., and Levitz, M.: Transport of amino acids by placenta. Amer. J. Obstet. Gynecol., 101: 820 (1968).

4. FaIn, J. N., ANd Scow, R. O.: Fatty acid synthesis in vivo in maternal and fetal tissues in the rat. Amer. J. Physiol., 210: 19 (1966).

5. Flexner, L. B., AND Pohl, H. A.: Transfer of radioactive sodium across the placenta of the guinea pig. Amer. J. Physiol., 132: 594 (1941).

6. Goldwater, W. H., and Stetten, D., JR.: Studies in fetal metabolism. J. Biol. Chem., 169: 723 (1949).

7. Herberc, R. J.: Determination of carbon-14 and tritium in blood and other whole tissues. Anal. Chem., 32: 42 (1960).

8. Herschfield, M. S., ANd Nemeth, A. M.: Placental transport of free palmitic and linoleic acid in guinea pig. J. Lipid Res., 9: 460 (1968).

9. Hirsch, J., Farquhar, J., Ahrens, E. H., Jr., Peterson, M. L., AND STOFfel, W.: Studies of adipose tissue in man, a microtechnic for sampling and analysis. Amer. J. Clin. Nutr., 8: 499 (1960).

10. Kayden, H. J., Dancis, J., And Money, W. L.: Transfer of lipids across the guinea pig placenta. Amer. J. Obstet. Gynecol., 104: 564 (1969).

11. Koren, Z., AND Shafrir, E.: Placental transfer of free fatty acids in the pregnant rat. Proc. Soc. Exp. Biol. Med., 116: 411 (1964).

12. Krantz, K. E., Panos, T. C., and Evans, J.: Physiology of maternal-fetal relationship through the extracorporeal circulation of the human placenta. Amer. J. Obstet. Gynecol. 83: 1214 (1962).

13. Levriz, M., AND Dancis, J.: Transfer of steroids between mother and fetus. Clin. Obstet. Gynecol., 6: 62 (1963).

14. Money, W. L., and Dancis, J.: Technique for the in situ study of placental transport using the pregnant guinea pig. Amer. J. Obstet. Gynecol., 80: 209 (1960).

15. Portman, O. W., Behriman, R. E., and Soltys, P.: Transfer of fatty acids across the primate placenta. Amer. J. Physiol., 216: 143 (1969).

16. Robertson, A., Sprecher, H., AND KarP, W.: Oxidation of palmitate by human placental tissue slices. Physiol. Chem. Phys., 3: 293 (1971).

17. Schneider, H., Panigel, M., and Dancis, J.: Transfer across perfused human placenta: sodium, antipyrine and leucine. Amer. J. Obstet. Gynecol., 114: 822 (1972).

18. Sheath, J., Grimwade, J., Waldron, K., Bickley, M., Taft, P., AND WOOD, C.: Arteriovenous nonesterified fatty acids and glycerol differences in the umbilical cord at term and their relationship to fetal metabolism. Amer. J. Obstet. Gynecol., 113: 358 (1972).

19. Szabo, A. J., Grimaldi, R. D., and Jung, W. F.: Palmitate transport across perfused human placenta. Metabolism, 18: 406 (1969).

20. VanDuyne, C. M., Havel, R. J., and Felts, J. M.: Placental transfer of palmitic acid-1. ${ }^{14} \mathrm{C}$ in rabbits. Amer. J. Obstet. Gynecol., 84: 1069 (1962).

21. Vanduyne, C. M., Parker, H. R., Havel, R. J., and Holm, L. W.: Free fatty acid metabolism in fetal and newborn sheep. Amer. J. Physiol., 199: 987 (1960).

22. Wrddowson, E. M., And Spray, C. M.: Chemical development in utero. Arch. Dis. Child., 26: 205 (1951).

23. Yoshioka, T., AND Roux, J. F.: In vitro metabolism of palmitic acid in human fetal tissue. Pediat. Res., 6: 675 (1972).

24. Sigma Chemical Company, St. Louis, Mo.

25. Tri-Carb model no. 3375, Packard Instrument Company, Inc., Downers Grove, Ill.

26. NCS, Amersham-Searle Corporation, Arlington Heights, Ill.

27. Analtech, Newark, Del.

28. New England Nuclear, Boston, Mass.

29. Schwarz-Mann, Orangeburg, N. Y.

30 . With the flow rates used in these studies, the maximal antipyrine clearance was $2.93 \mathrm{ml} / \mathrm{min}$ [17]. The assumption is made that the maximal clearance most closely approximates the in vivo situation.

31. See Reference 18.

32. Dr. Dancis is a Career Investigator, National Institutes of Health, Grant no. 5-K6-HD-16,710-10.

33. This research was supported by National Institutes of Health Grants no. 5-R01 HD00462 and 5 R01 CA02071-19 and United States Public Health Service Grant no. HEO 6481.

34. Dr. Schneider was supported by Deutsche Forschungsgemeinschaft.

35. Requests for reprints should be addressed to: JoSEPH DANCxs, M.D., Department of Pediatrics, New York University Medical Center, 550 First Avenue, New York 10016 (USA).

36. Accepted for publication December 11, 1972. 